\title{
JASPION: Jogo Sério Educacional Sensível ao Contexto Integrado ao Mundo Virtual OpenSim
}

\author{
Aliane L. Krassmann ${ }^{1}$, Leo Natan Paschoal ${ }^{2}$, Andressa Falcade ${ }^{1}$, Roseclea D. \\ Medina $^{1}$ \\ ${ }^{1}$ Programa de Pós-Graduação em Ciência da Computação - Universidade Federal de \\ Santa Maria (UFSM) - Santa Maria - RS - Brasil \\ ${ }^{2}$ Graduação em Ciência da Computação - Universidade de Cruz Alta (UNICRUZ) - \\ Cruz Alta - RS - Brasil \\ \{alkrassmann, leonatanpaschoal, andressafalcade, \\ roseclea.medina\} @gmail.com
}

\begin{abstract}
This study presents a proposal for the development of JASPION, an adaptable to the user context's serious game, integrated into a pre-existing virtual world, built on OpenSim platform, which offers computer networks discipline's educational objects from Computer Science higher education. It is intended to provide the student a gameplay profile personalized to his cognitive style and level of expertise, supporting a significant and versatile learning process by using serious games.
\end{abstract}

Resumo. Este estudo apresenta uma proposta para o desenvolvimento do JASPION, um jogo sério adaptável ao contexto do usuário, integrado a um mundo virtual pré-existente, construído na plataforma OpenSim, que dispõe de objetos educacionais da disciplina de redes de computadores do ensino superior em Ciência da Computação. Pretende-se propiciar ao estudante um perfil de jogabilidade personalizado ao seu estilo cognitivo e nível de expertise, apoiando um processo de aprendizagem significativa e versátil por meio da utilização de jogos sérios.

\section{Introdução}

O desenvolvimento de metaversos ou Mundos Virtuais (MVs), quando elaborados com o objetivo de apoiar o processo ensino-aprendizagem, vem propiciando aos educadores e educandos alternativas inovadoras neste campo. Ávila et al. (2013), descrevem em sua pesquisa que atividades baseadas em computador, quando elaboradas com propósitos claros, tendem a ensejar uma interação ativa do estudante com o objeto de conhecimento, ao mesmo tempo em que lhe proporciona autonomia para determinar o ritmo de seu processo de aprendizagem.

Os MVs permitem o envolvimento do aluno em contextos diversificados, com situações realísticas que são difíceis de serem trabalhadas na forma tradicional de ensino [Voss et al. 2013]. Propiciam ao estudante imersão às atividades do curso, com conteúdos e também com professor e colegas [Piovesan et al. 2013].

Nesse contexto também emergem os Jogos Sérios (JS), como ferramentas para motivar e melhorar o aprendizado, sendo apoiados por diversas pesquisas atuais, como 


\section{CBIE-LACLO 2015}

Anais do XXVI Simpósio Brasileiro de Informática na Educação (SBIE 2015)

as de Raman et al. (2014), Bordini et al. (2014) e Rodríguez-Cerezo et al. (2014). De acordo com Buchinger e Hounsell (2013) os JS possuem o objetivo principal e inicial de servir a um propósito sério, em especial à educação. Estão alinhados com as necessidades da geração atual, pois, conforme Prensky (2012), além de motivar pela diversão, são versáteis, podendo ser adaptados a diferentes disciplinas, informações ou habilidades a serem aprendidas.

O foco deste trabalho é descrever a proposta de um JS denominado JASPION (Jogo Sério Educacional Sensível ao Contexto Integrado ao Mundo Virtual OpenSim), integrado a um MV pré-existente $\left(\mathrm{TCN}^{5}\right)$ implementado por Voss (2014), que adapta técnicas de computação consciente de contexto e dispõe de objetos educacionais da disciplina de redes de computadores do ensino superior em Ciência da Computação. Pretende-se propiciar ao usuário um perfil de jogabilidade personalizado ao seu estilo cognitivo e nível de expertise, verificando se a abordagem é capaz de apoiar uma aprendizagem significativa e versátil.

Esta pesquisa é embasada nos princípios da Teoria da Flexibilidade Cognitiva (TFC) de Spiro et al. (1990, 2006), e da Aprendizagem Significativa, de Ausubel (2000), que envolvem a aquisição de novos significados de forma agradável, que aguça a curiosidade intelectual e a perspectiva de adquirir novos conhecimentos, em vez de provocar uma reação como se fosse uma tarefa não recompensada e desagradável.

\section{Jogos Sérios e Mundos Virtuais na Educação}

De acordo com Chen (2011), espaços virtuais, como jogos e mundos virtuais, permitem abstrações da realidade que não só incentivam a aprendizagem auto-dirigida do educando, mas também reforçam o conteúdo central do objetivo de aprendizagem através de metáforas visuais não reprodutíveis no mundo físico.

Segundo Salen e Zimmerman (2012), um jogo pode ser definido como um sistema no qual os jogadores se envolvem em um conflito artificial, definido por regras, que implica em um resultado quantificável. Assim, o encorajamento provocado pelos JS vai exigir que o participante explore, pense e repense, ou seja, faz com que reflita sobre suas decisões, na medida em que cada ação acarreta em uma futura reação no jogo, que poderá favorecer ou dificultar sua chegada ao objetivo [Reis et al. 2013].

Nos dias de hoje os MVs são cada vez mais utilizados para inúmeras finalidades, como proporcionar a motivação e aperfeiçoar o aprendizado dos usuários em ambientes educacionais. Ávila et al. (2013) destacam que características como possibilidade de colaboração, participação ativa do estudante, incentivo à investigação e à resolução de problemas, costumam ser inerentes aos laboratórios de aprendizagem que, com os avanços tecnológicos, vêm sendo oferecidos através dos ambientes virtuais.

Disciplinas que necessitam de um laboratório físico, como redes de computadores, podem causar um grande impacto devido ao custo e ao risco das ações que o usuário executa. Os metaversos, proporcionam a simulação de situações reais ou fictícias, reduzindo custos com aquisição e manutenção de laboratórios físicos [Voss et al. 2013]. Possibilitam exercitar conhecimentos práticos em cenários virtuais tridimensionais (3D), permitindo maior interatividade, tentativas e erros, respeitando seu tempo cognitivo individual e enfatizando a aplicação prática de seu aprendizado.Também podem ser utilizados como plataformas para o desenvolvimento de jogos. 


\section{Proposta do Jogo}

Esta proposta visa unir os benefícios dos jogos sérios às funcionalidades imersivas de um mundo virtual, propondo a criação de um jogo educacional ciente do contexto do usuário. O jogo será integrado a um MV OpenSim pré-existente, voltado ao ensino de redes de computadores [Voss 2014], para aplicação junto a alunos do curso superior em Ciência da Computação. Propõe-se que o estudante adquira uma aprendizagem significativa por meio de imersão, desafios e dinamicidade, componentes importantes da educação contemporânea, segundo Prensky (2012) e Ávila et al. (2013).

Visando evitar ou atenuar os sentimentos de desestimulação no acesso ao metaverso e, consequentemente, à persistência no jogo, que podem ocorrer devido à falta de relação ou identificação do estudante com o ambiente, evitando torná-lo apenas um produto didatizado, surge a necessidade de criação de um jogo sério ciente ao contexto do usuário, ou seja, que se adapta à sua realidade (estilo cognitivo e nível de expertise), tornando-o mais atraente e eficaz em sua proposta.

Pretende-se propiciar ao usuário um perfil de jogabilidade personalizado adequadamente a dois fatores, são eles:

1. Estilo cognitivo, que segundo Mozzaquatro (2010) está relacionado ao modo de percepção dos dados e à formulação de conhecimentos a partir dos dados assimilados, influenciando em atitudes, valores e interação social. Pode ser utilizado para indicar estratégias instrucionais mais eficazes para um dado indivíduo.

2. Nível de expertise, que de acordo com Possobom et al. (2014) refere-se à escala de conhecimento de um estudante sobre determinada área, levando em consideração seu conhecimento prévio. Pode fazer com que alunos não se desestimulem em realizar atividades que não estão de acordo com seu nível de conhecimento.

\subsection{Design do Jogo}

Conforme Trois (2013), o design de games é um conceito de atividade que se refere ao projeto de jogos, englobando os mais diversos aspectos do ciclo de desenvolvimento, no qual são tomadas as principais decisões. Nesse processo, são feitas definições como história, aparência, andamento do jogo, recursos, conflitos, punições e recompensas.

A proposta do jogo é centrada em uma temática que mistura aventura e ação, focando em um enredo com resolução de enigmas e permitindo ação do jogador (um avatar no MV) que é retratado na figura de um herói. O protagonista, como no título do jogo, é o personagem Jaspion (uma aglutinação das palavras Justice e Champion), da série de televisão japonesa autoentitulada, exibida no Brasil entre as décadas de 80 e 90, que ganhou grande popularidade mundial.

A narrativa do jogo gira em torno do personagem-herói Jaspion, que deve proteger o planeta Network, que alimenta o planeta Terra com o poder da Internet. O antagonista Satan Goss, do Império dos Monstros, deseja interromper esta conexão para conquistar o Universo. Para isso, em cada fase Jaspion encontra o planeta Network desconfigurado por Satan Goss em algum aspecto, como equipamentos de redes, topologia, definições de sistemas de rede, entre outros ativos que impedem ou dificultam o tráfego de rede.

O protagonista tem de reparar todo o estrago em um tempo estabelecido, identificando e corrigindo o que está desconfigurado ou alterado no planeta Network, para que volte a funcionar corretamente. A cada enigma solucionado, Jaspion agrega 
poderes, enquanto Satan Goss enfraquece. Da mesma forma o contrário ocorre, na medida em o tempo diminui, o antagonista fortalece-se, e, no seu auge, ao término do tempo delimitado para a fase, sofre uma metamorfose que o transforma em o Poderoso Satan Goss, criatura aberracional e imbatível.

Cada nível de dificuldade do jogo é composto por 5 fases sequenciais, de complexidades crescentes. A cada nível ultrapassado é apresentada uma versão diferente do antagonista, que fica mais monstruosa na medida em que a dificuldade aumenta. Ao chegar à última fase do último nível, Jaspion consegue então fazer frente a Satan Goss, com seu golpe fatal, salvando o planeta Network e protegendo a Terra contra a perda de conexão com a Internet.

\subsection{Mecânica do Jogo}

A mecânica do jogo é o que promove a interatividade dos elementos com o jogador, onde se encontram as regras e os objetivos, oferecendo opções, escolhas, permissões e proibições do jogo [Sato e Cardoso 2008]. Adotou-se a expressão "mecânica do jogo" ao invés do termo gameplay neste artigo, em concordância com Sato e Cardoso (2008), devido a não haver tradução direta para gameplay no Brasil.

O jogo é no formato single-player (um único jogador) e o objetivo consiste em responder aos desafios propostos, solucionando problemas de redes por meio da escolha entre opções apresentadas, de modo a avançar para as próximas fases até atingir o seu estágio final. Os scripts na linguagem OSSL (Open Simulator Scripting Language) são responsáveis por gerir regras, condições, premiações e demais ocorrências estabelecidas do jogo, bem como conduzir o seu funcionamento, definindo a parte relacionada à programação.

Devido à complexidade em criar a aparência do protagonista Jaspion e do antagonista Satan Goss, será utilizado o software Blender para modelar os avatares, exportando os arquivos na extensão ".dae". Entretanto alguns viewers não suportam a importação neste formato. Conforme Voss (2014), o viewer Singularity atende ao requisito de importação de arquivos com essa extensão, sendo então definido como ferramenta para acesso à interface gráfica do MV neste estudo.

No jogo, os usuários possuem três níveis de classificação de expertise, baseado em Possobom et al. (2014): básico (1), intermediário (2) e avançado (3). Além disso, possuem quatro classificações de estilo cognitivo, adaptados de Mozzaquatro (2010): (1) serialista, (2) holista, (3) reflexivo e (4) divergente. Sendo assim, há 12 perfis de jogabilidade, que surgem do cruzamento das duas classificações, conforme Tabela 1.

Tabela 1. Perfis de jogabilidade de acordo com nível de expertise e estilo cognitivo

\begin{tabular}{|c|c|c|}
\hline Perfil de jogabilidade & Nível de expertise & Estilo cognitivo \\
\hline $\mathbf{1}$ & Básico & Serialista \\
\hline $\mathbf{2}$ & Intermediário & Holista \\
\hline $\mathbf{3}$ & Avançado & Reflexivo \\
\hline $\mathbf{4}$ & Básico & Divergente \\
\hline $\mathbf{5}$ & Intermediário & Serialista \\
\hline $\mathbf{6}$ & Avançado & Holista \\
\hline $\mathbf{7}$ & Básico & Reflexivo \\
\hline
\end{tabular}


CBIE-LACLO 2015

Anais do XXVI Simpósio Brasileiro de Informática na Educação (SBIE 2015)

\begin{tabular}{|c|c|c|}
\hline $\mathbf{8}$ & Intermediário & Divergente \\
\hline $\mathbf{9}$ & Avançado & Serialista \\
\hline $\mathbf{1 0}$ & Básico & Holista \\
\hline $\mathbf{1 1}$ & Intermediário & Reflexivo \\
\hline $\mathbf{1 2}$ & Avançado & Divergente \\
\hline
\end{tabular}

Sendo assim, os três níveis de dificuldade correspondem aos três níveis de expertise. Assim, o jogador que completar o nível em que se encontra (composto de cinco fases), desbloqueia o acesso para o nível sequinte, podendo evoluir até o nível de avançado. Ao todo são 15 fases disponíveis no jogo (cinco para cada nível de dificuldade), sempre de acordo com o estilo cognitivo do usuário.

Como materiais de apoio para a realização das atividades, estão dispostos Presenters ao transcorrer do jogo, que contêm slides e vídeos sobre o conteúdo em questão, além de glossários para consulta dos usuários [Voss et al. 2013]. Os materiais didáticos de apoio seguem as preferências de estilo cognitivo do estudante, conforme Figura 1 (adaptado de Mozzaquatro (2010)).

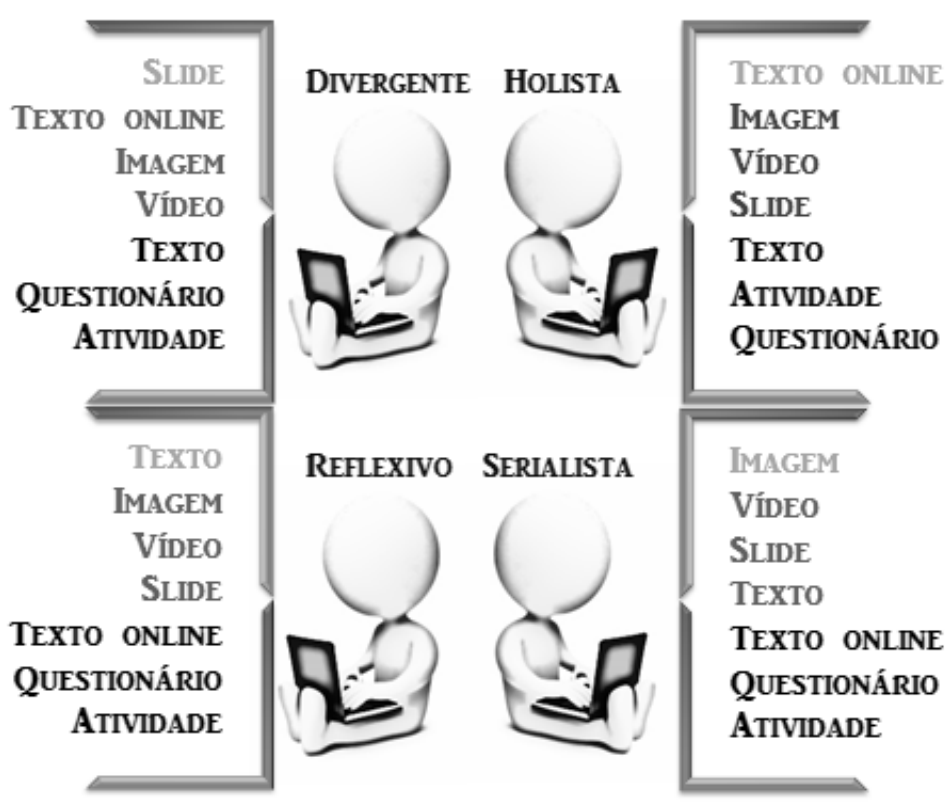

Figura 1. Materiais didáticos preferenciais por estilo cognitivo em ambientes virtuais (adaptado de Mozzaquatro (2010))

Na próxima seção, os procedimentos metodólogicos que orientam esta pesquisa são apresentados.

\section{Procedimentos Metodológicos}

A arquitetura do sistema apresenta a utilização de uma aplicação cliente-servidor, para acessar o jogo no mundo virtual remota ou localmente. Serão agregados os seguintes módulos ao JASPION (Figura 1):

1. Mundo Virtual $\mathrm{TCN}^{5}$ - Teaching Computer Networks In a Free Immersive Virtual Environment [Voss 2014];

2. Identificação do estilo cognitivo do usuário [Mozzaquatro 2010]; 
CBIE-LACLO 2015

Anais do XXVI Simpósio Brasileiro de Informática na Educação (SBIE 2015)

3. Identificação do nível de expertise do usuário [Possobom et al. 2014];

4. Desenvolvimento do jogo sério na plataforma OpenSim: em desenvolvimento.

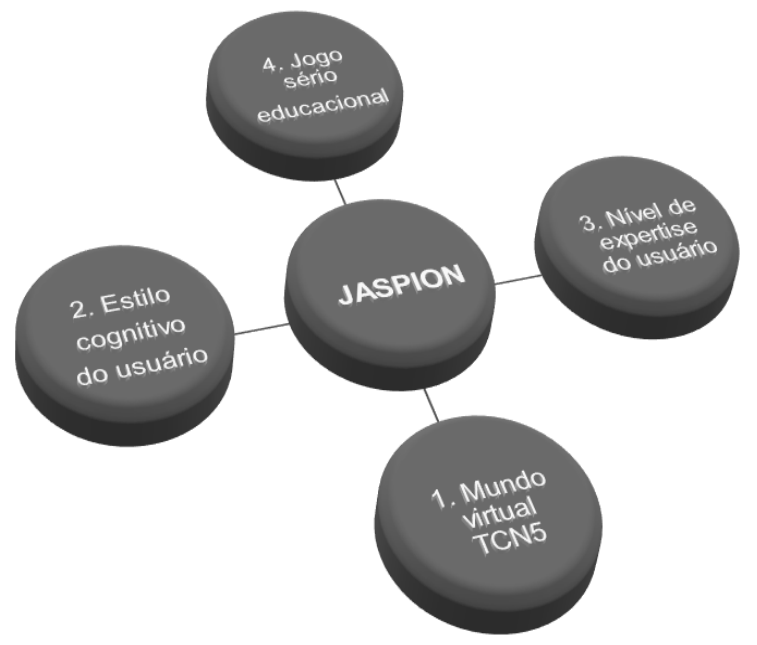

Figura 2. Módulos que integram o JASPION

$\mathrm{Na}$ construção deste estudo, são seguidas as etapas metodológicas conforme descritas abaixo.

$\mathrm{Na}$ primeira etapa é realizado um levantamento de literatura sobre os tópicos que envolvem o JASPION: aplicação e desenvolvimento de JS e MVs na educação, ensino de redes de computadores, computação consciente de contexto. Na segunda etapa são analisadas e testadas as ferramentas computacionais a serem utilizadas, como OpenSimulator, Singularity, Blender e Wampp, de modo a compreender suas principais funcionalidades para colocá-las em prática com eficiência.

$\mathrm{Na}$ terceira etapa é realizada a modelagem do jogo, considerando aspectos como definição do design e da mecânica do jogo, definição e adaptação do perfil de jogabilidade, criação das narrativas e desafios-enigmas correspondentes. Na quarta etapa é realizado o desenvolvimento do jogo, com a integração dos módulos préexistentes de detecção de contexto (Figura 2), criação dos scripts que envolvem a dinâmica do jogo, e realizações de testes e análises de desempenho.

Na quinta etapa o jogo é aplicado junto a um contexto real de estudantes, alunos do curso de Ciência da Computação. Após, é procedida a realização das avaliações de contexto computacional, via adaptação do questionário de usabilidade do SUS (System Usability Scale) (Brooke, 1986), e avaliação do contexto educacional via aplicação de pré e pós-testes, e de mapas conceituais (Novak e Cañas, 2008). Com a coleta de dados, será verificada a maneira com que o JASPION influenciou a proposta pedagógica da disciplina de redes de computadores e a cognição do estudante.

\section{Resultados Parciais}

Até o presente momento foram concluídas as etapas 1 e 2 da metodologia, tendo sido iniciada a terceira etapa.

A interação no jogo é realizada pelo protagonista Jaspion, um avatar controlado pelo estudante caracterizado com uma aparência semelhante ao personagem ao qual faz alusão. Desse modo, alguns testes já foram realizados, sendo um deles o da 


\section{CBIE-LACLO 2015}

Anais do XXVI Simpósio Brasileiro de Informática na Educação (SBIE 2015)

customização de aparência. Para desenvolvê-los utilizou-se o viewer Singularity (Figura $3)$.

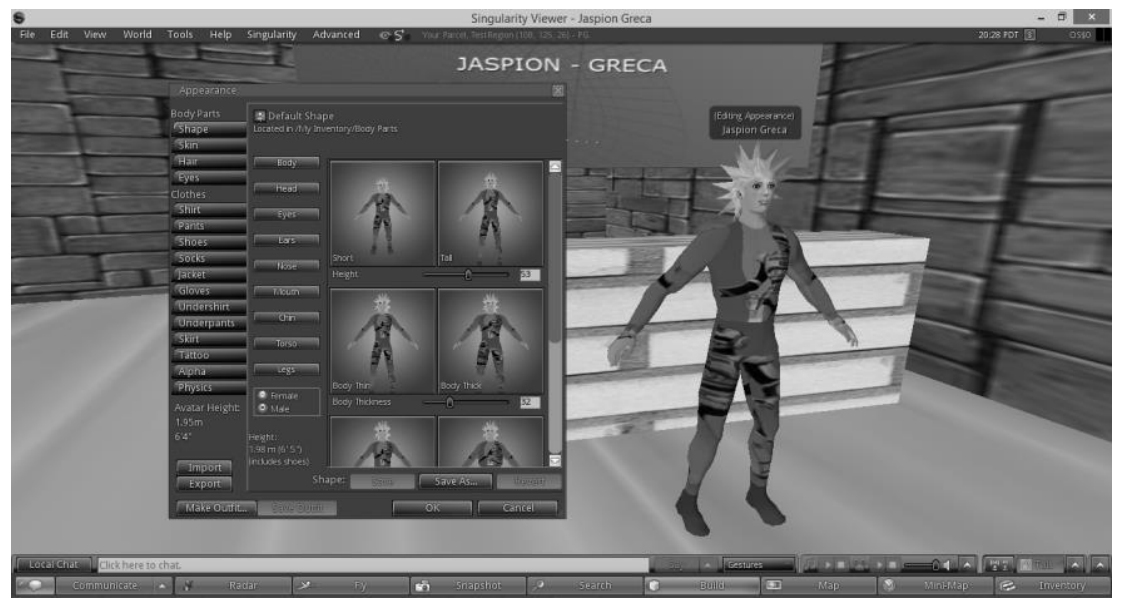

Figura 3. Editando a aparência do protagonista Jaspion no viewer Singularity

Para dar início ao desenvolvimento do jogo, algumas ferramentas de criação de objetos 3D para inserção nas fases foram testadas, o que elucidou a necessidade de disponibilização de material instrucional para os jogadores, para saberem como utilizar tais objetos. Percebeu-se, assim, a necessidade de realizar uma integração do MV com o ambiente Moodle, via plugin Sloodle, para que o usuário tenha acesso ao tutorial do jogo (manual de instruções), que fique disponível para consulta a qualquer momento de sua interação (Figura 4).

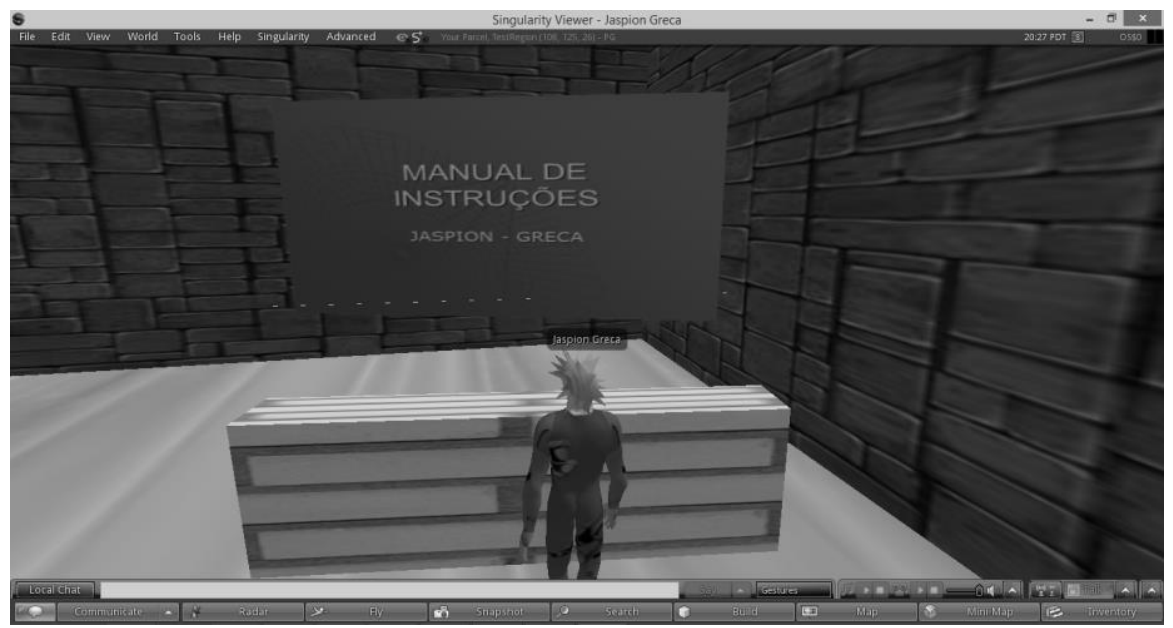

Figura 4. Jogador visualizando o manual de instruções via integração Sloodle/Moodle

Voss (2014) criou uma região para cada estilo cognitivo de aluno. Neste sentido o sistema proposto irá apropriar-se das regiões desenvolvidas e criar novas regiões para adaptar o $\mathrm{TCN}^{5}$ ao nível de expertise e estilo cognitivo, totalizando 12 regiões, de acordo com cada perfil de jogabilidade (Tabela 1). A Figura 5 ilustra o mapa das regiões desenvolvidas por Voss (2014). 


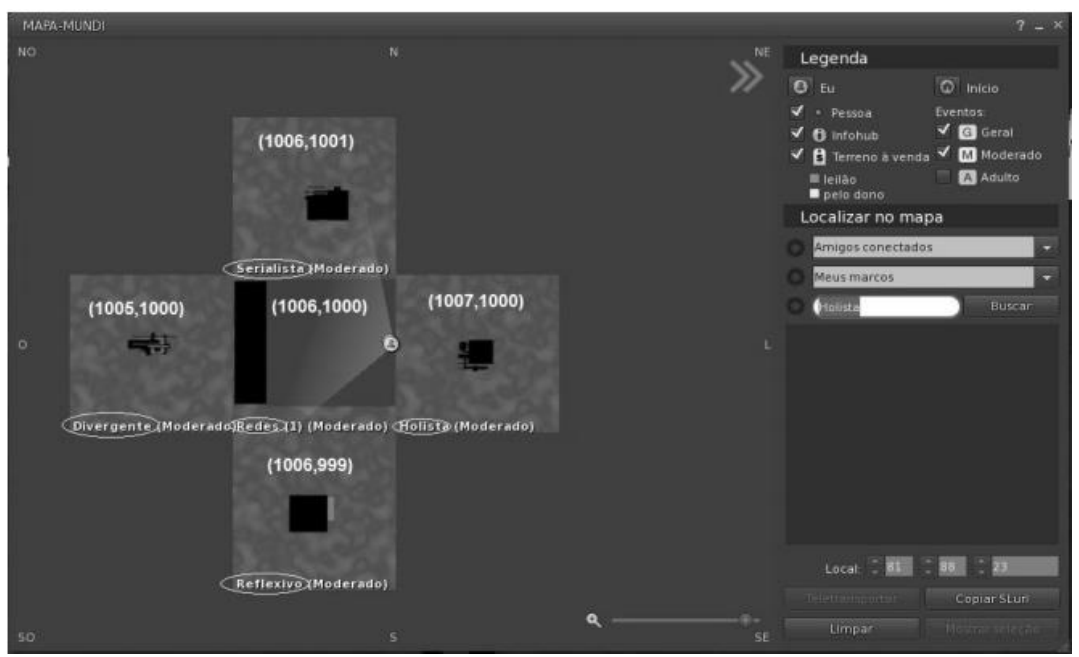

Figura 5. Mapa das regiões criadas no Mundo Virtual TCN ${ }^{5}$ (Voss, 2014)

Alguns resultados e impactos esperados com o desenvolvimento e finalização desta pesquisa são:

- $\quad$ Realização de um estudo de caso que comprove e valide a influência da proposta na cognição, planejando um percurso pedagógico para o estudante de redes de computadores;

- $\quad$ Provimento de um jogo sério consciente do contexto de aprendizagem do estudante, que adapte o conteúdo ao seu nível de expertise e estilo cognitivo, disponibilizando desafios e narrativas de acordo com seu perfil de jogabilidade;

- Integração a dispositivos móveis, possibilitando acesso ubíquo funcionalidades inovadoras ao processo ensino-aprendizagem;

- Disponibilização à comunidade acadêmica de uma ferramenta educacional interativa e dinâmica, que possibilite acesso ao conhecimento de forma ubíqua e personalizada, podendo ser adaptada para outros cursos/disciplinas.

\section{Considerações Finais}

O uso do paradigma de JS juntamente com os recursos de mundos virtuais criam a possibilidade dos usuários interagirem em um laboratório de redes de computadores com alto grau de imersão, permitindo a realização de atividades com elementos tanto da parte física quanto lógica, e que atividades de construção e configuração sejam desenvolvidas [Voss et al. 2013].

A pesquisa sobre jogos sérios na educação está em ascensão, intensificando-se nos últimos anos. Embora não seja uma plataforma destinada especificamente para o desenvolvimento de jogos, a tecnologia de mundos virtuais OpenSim permite que recursos de programação e de customização gráfica sejam implementados, possibilitando o desenvolvimento de jogos digitais 3D nesse background.

Esta pesquisa, denominada "JASPION: Jogo Sério Educacional Integrado a um Ambiente Virtual Imersivo Sensível ao Contexto do Usuário", visa desenvolver um jogo sério educacional integrado a um MV pré-existente, que dispõe de objetos educacionais do ensino formal superior em Ciência da Computação, construído em plataforma OpenSim. O diferencial do trabalho consiste no tratamento de informações de contexto do aprendiz (e.g., estilo cognitivo e nível de expertise), oferecendo assim uma 


\section{CBIE-LACLO 2015}

Anais do XXVI Simpósio Brasileiro de Informática na Educação (SBIE 2015)

experiência personalizada de jogabilidade e, por conseguinte, de potencial aprendizagem.

A proposta encontra-se atualmente em desenvolvimento. Com a sua finalização, pretende-se estimular o interesse dos alunos e verificar as influências cognitivas de jogos sérios decorrentes da abordagem aplicada, contribuindo para esta área do conhecimento tão complexa e volátil que é a Computação, especificamente no que se refere ao ensino de redes de computadores.

\section{Referências}

Ávila, Bárbara; Amaral, Érico M. H.; Tarouco, Liane. (2013). "Implementação de Laboratórios Virtuais no metaverso OpenSim." Revista Novas Tecnologias na Educação (Renote), v. 11, n1.

Ausubel, David P. (2000). "The acquisition and retention of knowledge: A cognitive view." Kluwer Academic Publishers.

Bordini, Rogério A.; Freitas, Pablo A. G.; Otsuka, Joice L.; Nunes, Antônio Pedro A.; Beder, Delano M.; Santiago, Daniel L.; Fonseca, Lucas F.; Santiago, Glauber L. A.; Oliveira, Marcia R. G. (2014). "Avaliação do Protótipo de um game educacional de Música.” Nuevas Ideas en Informática Educativa TISE, Fortaleza-CE, Brasil.

Brooke, J. (1986). "SUS: A "Quick and Dirty" Usability Scale". In P. W. Jordan, B. Thomas, B. A. Weerdmeester, \& A. L. McClelland. Usability Evaluation in Industry. London: Taylor and Francis.

Buchinger, D.; Hounsell, M. S. (2013). "Jogos Sérios Competitivo-Colaborativos: Um Mapeamento Sistemático da Literatura." II Congresso Brasileiro de Informática na Educação - CBIE. XXIV Simpósio Brasileiro de Informática na Educação - SBIE. DOI: 10.5753/CBIE.SBIE.2013.275 275.

Chen, Judy F.; Warden, Clyde A.; Tai, David Wen-Shung; Chen, Farn-Shing; Chao, Chich-Yang. (2011). "Level of abstraction and feelings of presence in virtual space: Business English negotiation in Open Wonderland." Computers \& Education 57 2126-2134, doi:10.1016/j.compedu.2011.05.017.

Mozzaquatro, Patricia Mariotto. (2010). "Adaptação do Mobile Learning Engine Moodle (Mle Moodle) aos Diferentes Estilos Cognitivos Utilizando Hipermídia Adaptativa." Dissertação de Mestrado. Programa de Pós-Graduação em Informática, Universidade Federal de Santa Maria, UFSM.

Novak, J. D. e Cañas, A. (2006). "The theory underlying concept maps and how to construct and use them." Technical Report IHMC CmapTools.

Possobom, Camila C.; Mülhbeier, Andreia R. K.; Nunes, Felipe B.; Carvalho, Aderson; Gomes, Ricardo B.; Medina, Roseclea D. (2014). "Uma Aplicação Dinâmica para Detectar o Nível de Conhecimento do Aluno.” XXIII Ciclo de Palestras Novas Tecnologias na Educação (CINTED), UFRGS.

Piovesan, S. D.; Wagner, R.; Medina, R. D.; Passerino, L. M. (2013). "Sistema Imersivo para Pessoas com Deficiência." XXIV Simpósio Brasileiro de Informática na Educação. 
Prensky, M. (2012). “Aprendizagem baseada em jogos digitais." São Paulo: Editora Senac, São Paulo, Brasil.

Raman, R.; Lal, A.; Achuthan, K. (2014). "Serious Games based approach to cyber security concept learning: Indian context." Green Computing Communication and Electrical Engineering (ICGCCEE).

Reis, Susana Cristina; Gomes, Adilson Fernandes; Panciera, Róger Junges; Menezes, Vitor Peixoto. (2013). "Da pesquisa à ação: conectando pressupostos teóricos e pedagógicos no desenvolvimento de um jogo de Inglês interdisciplinar em 3D." Nuevas Ideas en Informática Educativa TISE.

Rodríguez-Cerezo, D.; Sarasa-Cabezuelo, A.; Gómez-Albarrán, J. L. S. (2014). “Serious games in tertiary education: A case study concerning the comprehension of basic concepts in computer language implementation courses." Computers in Human Behavior, 3, p. 558-570.

Salen, K.; Zimmerman, E. (2012). "Regras do jogo: fundamentos do design de jogos." São Paulo: Blucher, Brazil.

Sato, Adriana K. O. e Cardoso, Marcos V. (2008). "Além do gênero: uma possibilidade para a classificação de jogos.” SBC - Proceedings of SBGames: Art \& Design Track.

Spiro, R. J.; Jehng, J. (1990). "Cognitive Flexibility and Hypertext: Theory and Technology for the nonlinear and multidimensional Traversal of Complex Subject Matter.” In: NIX, D.; SPIRO, R. J. (Eds.), Cognition, education, and multimedia. Hillsdale: Lawrence Erlbaum Associates, p.163-205.

Spiro, R. J.; Collins, B. P.; Ramchandran, A. R. (2006). "Modes of openness and flexibility in Cognitive Flexibility Hypertext learning environments." In: B. Khan (Ed.), Flexible learning in an information society. Hershey: Information Science Publishing, p. 18-25.

Trois, Sonia. (2013). "Propostas de Heurísticas para Adaptação de Conteúdo Instrucional a Games Educativos. Dissertação de Mestrado." Programa de PósGraduação em Design. Universidade Federal do Rio Grande do Sul (UFRGS).

Voss, G. B. (2014). "TCN ${ }^{5}$ - Teaching Computer Networks In a Free Immersive Virtual Environment." Dissertação de Mestrado, Universidade Federal de Santa Maria (UFSM), 159p.

Voss, G. B.; Oliveira, V.; Nunes, F.B.; Herpich, F.; Medina, R. D.; Bercht, M. (2013). "Construção e Análise de um Mundo Virtual 3D para o ensino e Aprendizagem de Redes de Computadores.” XXV Simpósio Brasileiro de Informática na Educação. 